\title{
Design and Optimization of Pyroelectric-Polymer Composites for Infrared Sensing and Energy Harvesting
}

\author{
Alakananda Bandyopadhyay ${ }^{1}$, Ashok Batra ${ }^{2 *}$, George Taylor ${ }^{2}$, Sharvare Palwai' \\ ${ }^{1}$ Department of Electrical Engineering and Computer Science, College of Engineering, Technology and Physical Sciences, \\ Alabama A \& M University, Normal (Huntsville), Alabama, USA \\ ${ }^{2}$ Materials Science Group, Department of Physics, Chemistry, and Mathematics, College of Engineering, Technology and \\ Physical Sciences, Alabama A \& M University, Normal (Huntsville), Alabama, USA \\ Email:*ashok.batra@aamu.edu
}

How to cite this paper: Bandyopadhyay, A., Batra, A., Taylor, G. and Palwai, S. (2020) Design and Optimization of PyroelectricPolymer Composites for Infrared Sensing and Energy Harvesting. Open Access Library Journal, 7: e6019.

https://doi.org/10.4236/oalib.1106019

Received: December 23, 2019

Accepted: January 3, 2020

Published: January 6, 2020

Copyright $\odot 2020$ by author(s) and Open Access Library Inc.

This work is licensed under the Creative Commons Attribution International License (CC BY 4.0).

http://creativecommons.org/licenses/by/4.0/

\begin{abstract}
In this paper, efforts have been made to investigate, and compute general formulae of the pyroelectric coefficient and figure-of-merit of pyroelectric-polymer composite for uncooled infrared detector and energy harvesting, using volume fraction, depolarization coefficient of particles and dielectric constants of the constituents.
\end{abstract}

\section{Subject Areas}

Fundamentals of Material Science

\section{Keywords}

Pyroelectric-Polymer

\section{Introduction}

Uncooled infrared (IR) detectors have rapidly advanced scientifically and technologically in recent years. In particular, IR sensors operating at room temperature are useful to many civilian and military applications, such as night vision, detection of gas leakages, surveillance, fire rescue operations, ear thermometer, manufacturing quality control, early fire detection and guidance, medical diagnostics, missile tracking, and an interception. There are two types of IR detectors: photon and thermal. In general, photon types are preferred primarily due to their superior sensitivity and resolution. However, the photon sensors must be cryogenically cooled down during operation to obtain high sensitivity which 
is a bulky and expensive proposition. Uncooled thermal sensors based on pyroelectric materials have attracted much attention because they operate at room temperature. These devices offer the advantage of low-cost fabrication and broadband of $8-14 \mu \mathrm{m}$ and beyond, In particular, in consumer applications such as human detection, burglar alarms, and door openers. The candidate materials for uncooled pyroelectric infrared detectors (PIR) are triglycine sulfate (TGS), lithium niobate (LN), lithium tantalate (LT), lead titanate (PT), lead zirconate titanate (PZT), lead strontium Titanate (PST), polyvinylidene fluoride (PVDF), polyvinylidene fluoride-trifluoroethylene copolymer ( $\mathrm{P}(\mathrm{VDF}-\mathrm{TrFE}))$, and others [1] [2] [3] [4]. PVDF is flexible and mechanically strong, has several attractive pyroelectric, ferroelectric, and piezoelectric properties with the reasonably fast, dynamic response for use in touch/tactile sensors, infrared detectors and vidicon/imaging devices [3] [4].

Pyroelectric: Polymer's composites can be considered an established substitute for conventional electro-ceramics and to pyroelectric polymers. The composites have a unique blend of polymeric properties such as mechanical flexibility, high strength, formability, and low cost with high electro-active properties of ceramic materials. It has attracted considerable interest because of their potential use in pyroelectric infrared detecting devices and piezoelectric transducers.

In the light of many technologically important applications in this field; it is worthwhile to investigate, and derive general formulae of the pyroelectric coefficient and figure-of-merit for uncooled infrared detector and energy harvesting, using volume fraction, depolarization coefficient of particles and dielectric constants of the constituents.

\section{Theoretical}

\subsection{Mathematical Modeling}

In the current study, a composite structure of continuous matrix (referred to as substance: 1) with the dielectric constant of $\epsilon_{1}$ and particles (referred to as substance; 2) with a dielectric constant $\epsilon_{2}$ has been considered. The pyroelectric particles are considered to be in ellipsoid shapes and distributed uniformly in the matrix. Also assumed that the volume of each of the ellipsoidal particle being same.

A few terms are defined here prior to the main mathematical equations used for deriving the composite dielectric constant, pyroelectric coefficient, and other characteristic parameters

$P=$ Polarization of the composite;

$P_{2}=$ Polarization of the ellipsoid particles;

$n_{x}=$ Depolarization coefficient of the ellipsoid particles in the $x$-direction;

$q=$ volume fraction which is defined as $=N^{*} V / V$ where $N$ is number of ellipsoid particles in a certain volume of the composite $(V)$ and volume of each ellipsoid particle is $v$;

$\alpha=$ Polarization ratio of the pyroelectric material.

The pyroelectric coefficient of the composite and ellipsoid is defined as: 


$$
\begin{gathered}
p_{c}=\mathrm{d} P / \mathrm{d} T \\
p_{2}=\mathrm{d} P_{2} / \mathrm{d} T
\end{gathered}
$$

The effective dielectric constant of the composite has been developed by Wang et al. [5], based on Clausius-Mossotti relation as:

$$
\epsilon_{c}=\epsilon_{1}\left[1+\frac{q\left(\epsilon_{2}-\epsilon_{1}\right)}{\epsilon_{1}+\left(\epsilon_{2}-\epsilon_{1}\right)(1-q) n_{x}}\right]
$$

The pyroelectric coefficient of the composite can be expressed as a function of the dielectric constant of the composite, pyroelectric coefficient of the ellipsoid particles $\left(p_{2}\right)$ as given below:

$$
p_{c}=\frac{\alpha q \epsilon_{c} p_{2}}{\epsilon_{c}+\left(\epsilon_{2}-\epsilon_{1}\right) n_{x}}
$$

The above two equations can be algebraically rewritten as a ratio of: 1) dielectric constant of composite with that of the particles and 2) pyroelectric coefficient to the dielectric constant of the composite as a function of a few dimensionless parameters as given below.

$$
\begin{gathered}
\frac{\epsilon_{c}}{\epsilon_{2}}=\frac{1}{\beta}\left[1+\frac{q(\beta-1)}{1+(\beta-1)(1-q) n_{x}}\right] \\
\frac{p_{c}}{\epsilon_{c}}=\frac{\alpha q}{\left(1-n_{x}\right) \frac{\epsilon_{c}}{\epsilon_{2}}+n_{x}} \frac{p_{2}}{\epsilon_{2}}
\end{gathered}
$$

The factor $\beta$ is defined as $\epsilon_{2} / \epsilon_{1}$.

\subsection{Figure-of-Merit}

The term figure-of-merit is used widely to characterize the performance of a device and it can be expressed in many different ways for a sensor. In the section below, a few different figures of merit are defined as below, which will be used for making the current analysis.

$$
\mathrm{FOM}=\frac{\frac{p_{c}}{\epsilon_{c}}}{\frac{p_{2}}{\epsilon_{2}}}=\frac{\alpha q}{\left(1-n_{x}\right) \frac{\epsilon_{c}}{\epsilon_{2}}+n_{x}}
$$

where the ratio of $\epsilon_{c} / \epsilon_{2}$ is given by Equation (3).

As can be observed from the above equation (Equation (5)), the figure of merit is a function only of the following four dimensionless parameters:

1) $\alpha$

2) $q$

3) $n_{x}$

4) $\beta\left(=\epsilon_{2} / \epsilon_{1}\right)$

The other of the figure-of-merit of device application for the current study are defined as below: 


$$
\begin{aligned}
& \mathrm{FOM}_{\mathrm{V}}=\frac{p_{c}}{\epsilon_{c}} \text { Figure-of-Merit for high voltage responsivity } \\
& \mathrm{FOM}_{\mathrm{VID}}=\frac{p_{c}}{\sqrt{\epsilon_{c}}} \text { Figure-of-Merit for vidicons } \\
& \mathrm{FOM}_{\mathrm{EH}}=\frac{p_{c}^{2}}{\epsilon_{c}} \text { Figure-of-Merit for thermal energy harvesting }
\end{aligned}
$$

\section{Results}

Figure 1 shows the dependence of relative figure-of-merit (FOM) on the volume fraction $q$ at different depolarization coefficient $n_{x}$ when $\epsilon_{2} / \epsilon_{1}=50$. It can be inferred that at an appropriate volume fraction, the FOM of the composite can be much higher than that of pure ferroelectric ceramic particles, even when $q$ is small, such as $q=0.05,0.15,0.3$. However, at some volume fraction $q$, the FOM can be much different for composites of different $n_{x}$ as illustrated in Figure 2 .

Different processing procedures may result in the different values of FOM. Figure 3 shows the dependence of FOM on relative dielectric constants $\epsilon_{2} / \epsilon_{1}$ at different volume fractions when $n_{x}=1 / 3$. It can be seen that FOM increases rapidly when $\epsilon_{2} / \epsilon_{1}$ is small, the increase becomes slower when $\epsilon_{2} / \epsilon_{1}$ exceeds a specific value. Thus, it can be inferred from the above-cited modeling results that the FOM of the composite not only depends on the volume fraction $q$, but it also depends on $n_{x}$ and the relative dielectric constants $\epsilon_{2} / \epsilon_{1}$. Figure 4 shows the optimized values of volume fraction $q$ and the depolarization coefficient $n_{x}$ to obtain highest value of FOM as a function of relative dielectric constant $\epsilon_{2} / \epsilon_{1}$.

Figure 5 shows the figure-of-merit for high voltage responsivity $\left(\mathrm{FOM}_{\mathrm{V}}\right)$ as a function of volume fraction of ferroelectric particles $q$ at different values of $n_{x}$. Figure 6 presents the variation of the figure-of-merit for vidicons $\left(\mathrm{FOM}_{\mathrm{VID}}\right)$ applications as the function of $q$ at various values of $n_{x}$. Figure 7 illustrates the figure-of-merit for thermal energy conversion $\left(\mathrm{FOM}_{\mathrm{EH}}\right)$ with $q$ as various values of $n_{x}$.

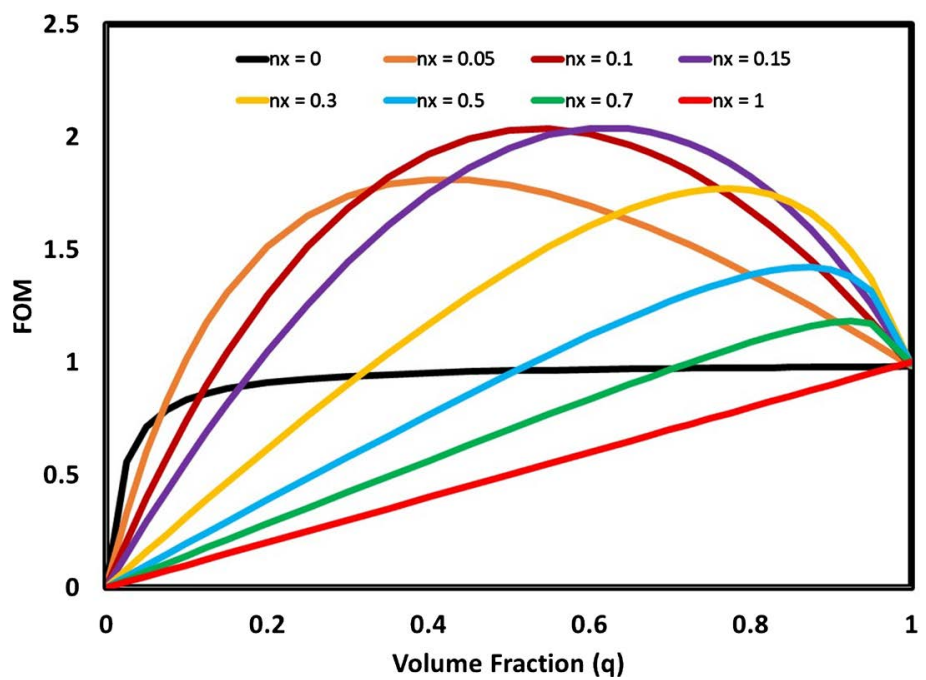

Figure 1. A figure-of-merit $\left(p_{c} / E_{d} / p_{2} / E_{2}\right)$ as a function of volume fraction $(q)$ at different $n_{x}$ 


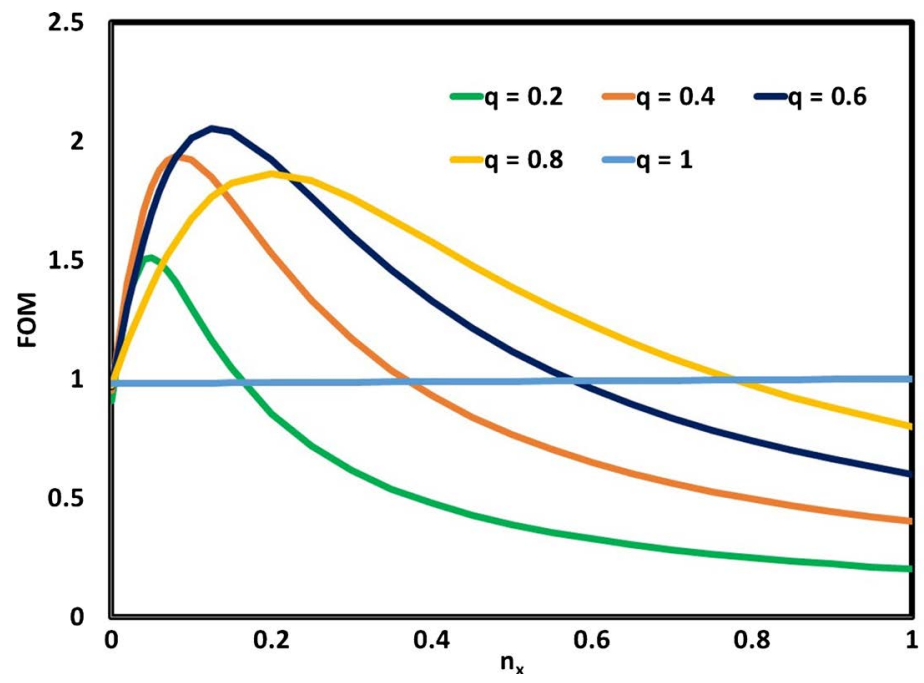

Figure 2. A plot of figure-of-merit as a function of $n_{x}$ at a different volume fraction.

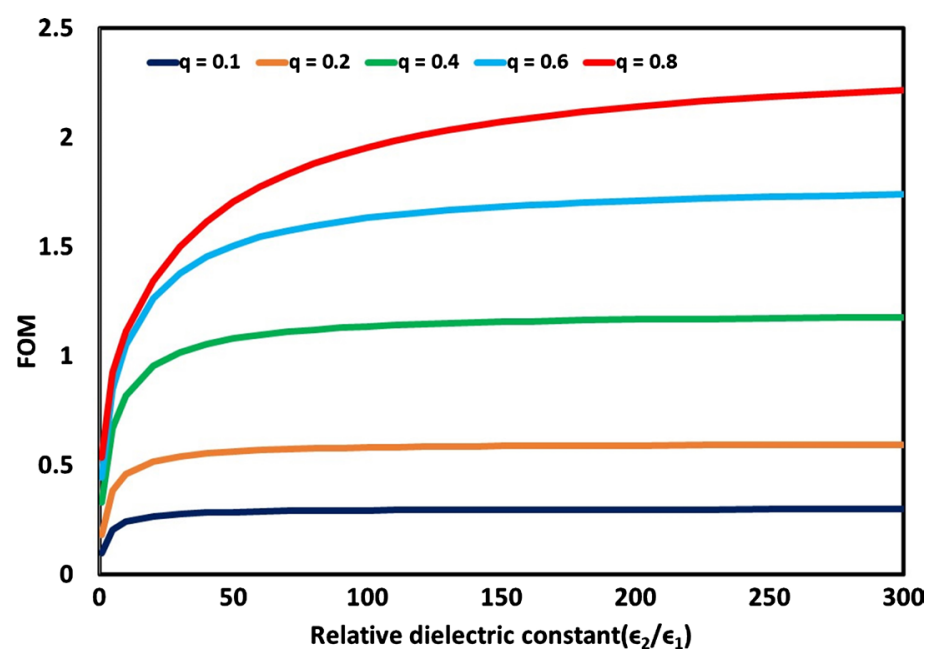

Figure 3. A plot of figure-of-merit as a function of relative dielectric constant $\left(\epsilon_{2} / \epsilon_{1}\right)$.

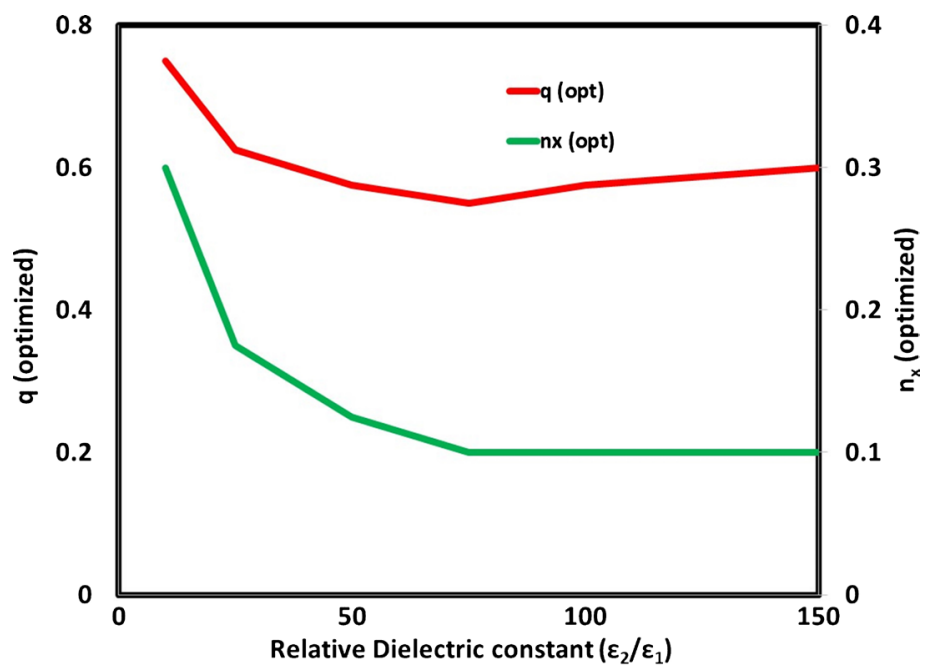

Figure 4. Optimized values of volume fraction and $n_{X}$ (along the secondary axis) to reach the maximum figure-of-merit as a function of $\epsilon_{2} / \epsilon_{1}$. 


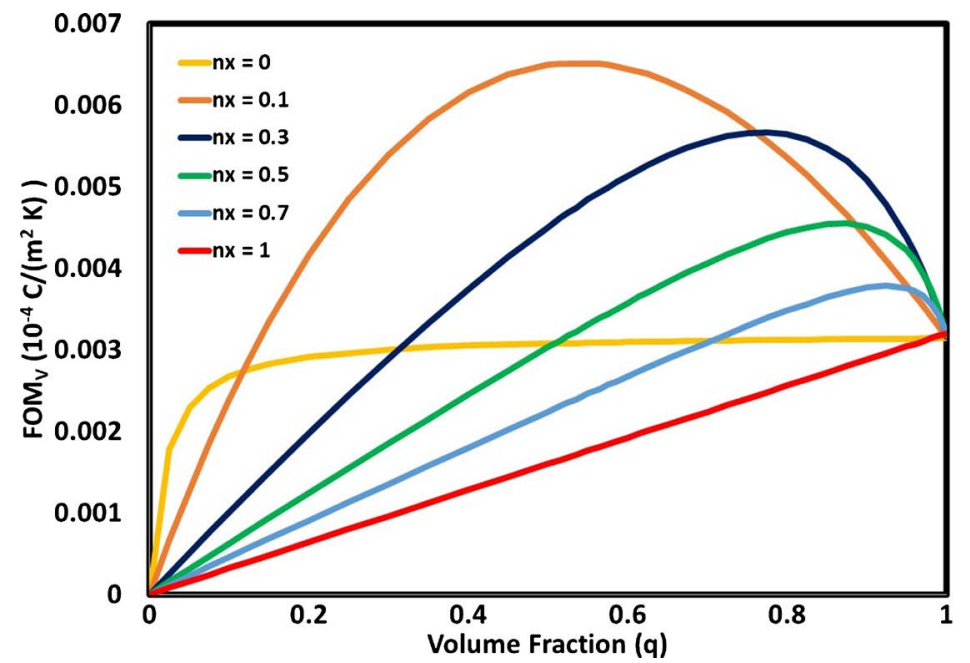

Figure 5. The figure-of-merit $\left(\mathrm{FOM}_{\mathrm{V}}\right)$ as a function of volume fraction at different $n_{x}$.

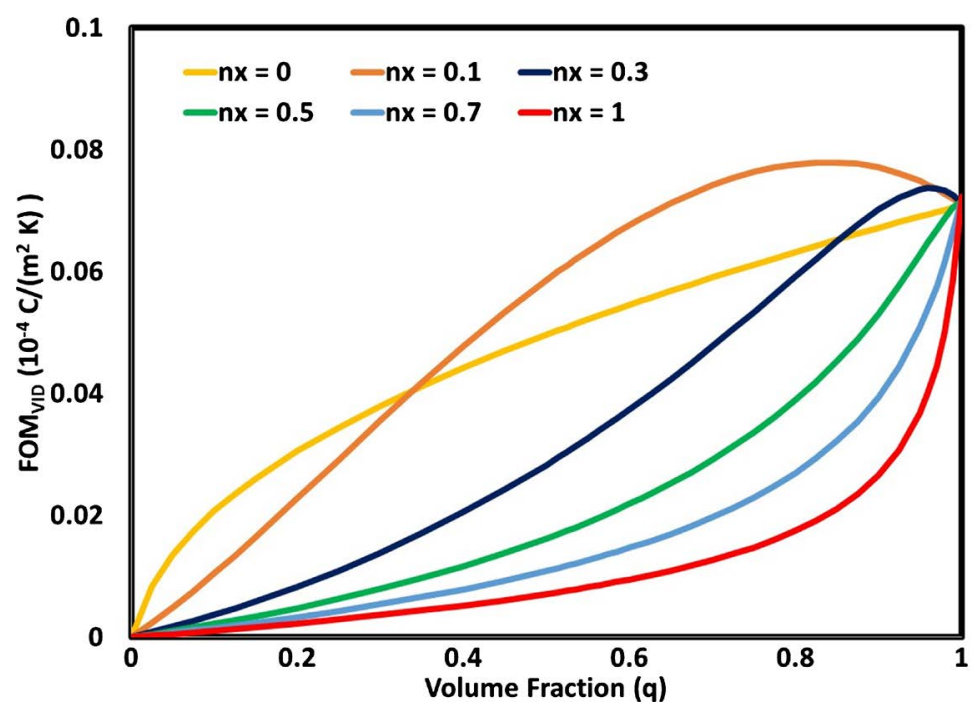

Figure 6. Figure-of-merit $\left(\mathrm{FOM}_{\mathrm{VID}}\right)$ as a function of volume fraction $(q)$ at different $n_{x}$.

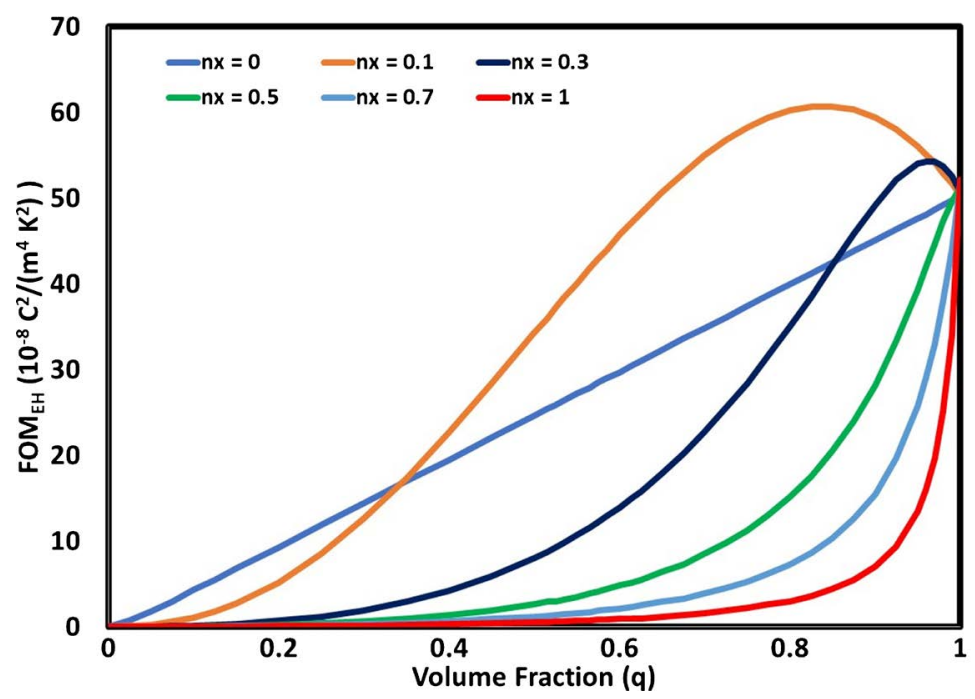

Figure 7. The figure-of-merit $\left(\mathrm{FOM}_{\mathrm{EH}}\right)$ as a function of volume fraction $(q)$ at different $n_{x}$. 
It is evident that for different applications of the composite pyroelectric detector, one has to select appropriate $n_{x}$ and $q$ so as to obtain maximum performance.

\section{Conclusion}

The important figure-of-merit of pyroelectric-polymer composite is computed in terms of the two components $\left(n_{x}\right.$ and $q$ ). They not only depend on the make-up of the composite, but also on the depolarization coefficient of the ferroelectric particles and relative dielectric constant $\epsilon_{2} / \epsilon_{1}$. The figure-of-merit of the composites can be higher than individual constituents. It is worth mentioning that different three parameters $\left(n_{x}, q, \epsilon_{2} / \epsilon_{1}\right)$ have to be optimized in order to obtain the maximum performance of the application device function.

\section{Acknowledgements}

The authors gratefully acknowledge the support of this work through the TitleIII program of AAMU.

\section{Conflicts of Interest}

The authors declare no conflicts of interest regarding the publication of this paper.

\section{References}

[1] Nalwa, H.S. (1995) Ferroelectric Polymers. Marcel Dekker, New York. https://doi.org/10.1201/9781482295450

[2] Batra, A.K., Aggarwal, M.D., Edwards, M.E. and Bhalla, A. (2008) Present Status of Polymer: Ceramic Composites for Pyroelectric Infrared Detectors. Ferroelectrics, 366, 84-121. https://doi.org/10.1080/00150190802363207

[3] Batra, A.K. and Alomari, A. (2017) Power Harvesting via Smart Materials. SPIE Press, Bellingham. https://doi.org/10.1117/3.2268643

[4] Batra, A.K. and Aggarwal, M.D. (2013) Pyroelectric Materials. SPIE Press, Bellingham.

[5] Wang, Y., Zhong, W., and Zhang, P. (1993) Pyroelectric Properties of Ferroelectric-Polymer Composite. Journal of Applied Physics, 74, 521-524. https://doi.org/10.1063/1.355263 